\title{
Regarding light resonance interpretations of the $B$ decay anomalies
}

\author{
Fady Bishara, ${ }^{1, *}$ Ulrich Haisch, ${ }^{1,2, \dagger}$ and Pier Francesco Monni ${ }^{2, *}$ \\ ${ }^{1}$ Rudolf Peierls Centre for Theoretical Physics, University of Oxford, OXI 3NP Oxford, United Kingdom \\ ${ }^{2}$ CERN, Theoretical Physics Department, CH-1211 Geneva 23, Switzerland
}

(Received 20 June 2017; published 5 September 2017)

\begin{abstract}
We sketch a novel method to search for light di-leptonic resonances by exploiting precision measurements of Drell-Yan production. Motivated by the recent hints of lepton flavor universality violation in $B \rightarrow K^{*} \ell^{+} \ell^{-}$, we illustrate our proposal by studying the case of spin-1 resonances that couple to muons and have masses in the range of a few GeV. We show that the existing LHC data on $p p \rightarrow$ $Z / \gamma^{*} \rightarrow \mu^{+} \mu^{-}$put nontrivial constraints on light di-muon resonance interpretations of $B$ decay anomalies in a model-independent fashion. The impact of our proposal on the long-standing discrepancy in the anomalous magnetic moment of the muon is also briefly discussed.
\end{abstract}

DOI: $10.1103 /$ PhysRevD.96.055002

\section{INTRODUCTION}

In the past four years several anomalies have been observed in rare semileptonic $B$ decays governed by $b \rightarrow s \ell^{+} \ell^{-}$transitions. Specifically, deviations from the standard model (SM) expectations in the angular observable $P_{5}^{\prime}$ [1] in $B \rightarrow K^{*} \mu^{+} \mu^{-}$[2-5], the branching ratios of $B^{+} \rightarrow K^{(*)+} \mu^{+} \mu^{-} \quad[6], \quad B \rightarrow K^{(*)} \mu^{+} \mu^{-} \quad[6,7]$ and $B_{s} \rightarrow \phi \mu^{+} \mu^{-}$[8] as well as the ratio $R_{K}$ [9] of di-muon to di-electron rates in $B^{+} \rightarrow K^{+} \ell^{+} \ell^{-}$[10] have been reported. The recent measurement of the ratio $R_{K^{*}}$ of dimuon to di-electron rates in $B \rightarrow K^{*} \ell^{+} \ell^{-}$has added to the list of anomalies [11] and has, accordingly, caught the attention of the theory community [12-30].

Although each deviation by itself is not statistically significant, and the angular observables and branching ratios are afflicted by hadronic uncertainties that obscure the interpretation and significance of the anomalies, it is quite intriguing that the deviations seen in the theoretically clean lepton-universality ratios $R_{K}$ and $R_{K^{*}}$ might be part of a coherent picture [12-14,16,17] involving new physics in the $b \rightarrow s \mu^{+} \mu^{-}$transitions in the form of the two dimensionsix operators $Q_{9}=\left(\bar{s}_{L} \gamma_{\alpha} b_{L}\right)\left(\bar{\mu} \gamma^{\alpha} \mu\right)$ and $Q_{10}=\left(\bar{s}_{L} \gamma_{\alpha} b_{L}\right)$ $\left(\bar{\mu} \gamma^{\alpha} \gamma_{5} \mu\right)$.

The most popular new-physics interpretations that can accommodate the $b \rightarrow s \ell^{+} \ell^{-}$anomalies involve new heavy degrees of freedom such as $Z^{\prime}$ bosons or leptoquarks (see e.g. [14] and references therein). Solutions that involve a new light resonance have instead received less

\footnotetext{
*fady.bishara@physics.ox.ac.uk ulrich.haisch@physics.ox.ac.uk *pier.monni@cern.ch
}

Published by the American Physical Society under the terms of the Creative Commons Attribution 4.0 International license. Further distribution of this work must maintain attribution to the author(s) and the published article's title, journal citation, and DOI. attention $[22,23,26,31,32],{ }^{1}$ although they might offer an explanation of the long-standing discrepancy (cf. [33]) in the anomalous magnetic moment of the muon $a_{\mu}=$ $((g-2) / 2)_{\mu}$. In fact, it has been shown very recently [22] that a spin-1 resonance with a mass of around $2.5 \mathrm{GeV}$ and a large invisible branching ratio can simultaneously explain both the flavor anomalies and $a_{\mu}$ while evading various other constraints, if the couplings of the mediator to fermions are dialed correctly.

In this paper, we point out that light resonance interpretations of the $b \rightarrow s \ell^{+} \ell^{-}$anomalies can be tested and constrained through precision studies of Drell-Yan (DY) production. ${ }^{2}$ Our finding is based on the simple observation that final state radiation (FSR) of a light di-leptonic resonance in $p p \rightarrow Z / \gamma^{*} \rightarrow \ell^{+} \ell^{-}$will lead to observable modifications of the kinematic distributions of the $\ell^{+} \ell^{-}$ system. We will illustrate this general idea by setting limits on the muon couplings of spin-1 resonances with masses in the $\mathrm{GeV}$ range by exploiting existing LHC data on the di-muon invariant mass $m_{\mu \mu}$ close to the $Z$ peak. The impact of this novel model-independent bounds on spin-1 mediator interpretations of the anomalies observed in rare semileptonic $B$ decays as well as $a_{\mu}$ will be discussed in some detail.

\section{SIMPLIFIED MODEL}

Following [22] we consider a simplified model valid at $\mathrm{GeV}$ energies which, besides the SM particles, contains a colorless spin-1 mediator $V$ with mass $m_{V}$ and a SM singlet

\footnotetext{
${ }^{1}$ The possibility that a light resonance could be responsible for the anomaly in $P_{5}^{\prime}$ was mentioned by Amarjit Soni at 50th Rencontres de Moriond EW 2015, and subsequently reemphasized to one of the authors by Brian Batell in a private conversation.

${ }^{2}$ Constraints on heavy di-lepton resonance interpretations of the $b \rightarrow s \ell^{+} \ell^{-}$tensions using present and future $p p \rightarrow \ell^{+} \ell^{-}$ data have been very recently derived in [28].
} 
Dirac fermion $\chi$ of mass $m_{\chi}$. The interactions of $V$ relevant for the further discussion are

$\mathcal{L} \supset\left(g_{L}^{s b} \bar{s}_{L} V b_{L}+\right.$ H.c. $)+\bar{\mu}\left(g_{V}^{\mu}-g_{A}^{\mu} \gamma_{5}\right) \forall \mu+g_{V}^{\chi} \bar{\chi} \nabla \chi$,

where, for concreteness, the couplings $g_{L}^{s b}, g_{V}^{\mu}, g_{A}^{\mu}$ and $g_{V}^{\chi}$ are taken to be real, $V=\gamma_{\alpha} V^{\alpha}$ and the subscript $L$ denotes left-handed fermionic fields. In what follows we will assume that $g_{L}^{s b}, g_{V}^{\mu}, g_{A}^{\mu}$ and $g_{V}^{\chi}$ encode all couplings between the new spin-1 state $V$ and fermions, and we will not specify an explicit ultraviolet completion that gives rise to them. We however add that the interactions (1) can emerge in various ways such as in vectorlike fermion extensions or by considering an effective approach where all $V$ couplings are generated via higher-dimensional operators (see e.g. [34,35]).

As demonstrated in [22], to qualitatively reproduce the $P_{5}^{\prime}, R_{K}$, and $R_{K^{*}}$ anomalies, the mass of the new spin-1 mediator is constrained to lie in the range of about $2-3 \mathrm{GeV}$ and its total decay width has to satisfy $\Gamma_{V} / m_{V} \gtrsim 10 \%$. The total width requirement implies that $m_{\chi}<m_{V} / 2$ and $g_{\chi} \gtrsim 2$. Consequently, $V$ predominantly decays invisibly with a branching ratio $\operatorname{Br}(V \rightarrow \bar{\chi} \chi) \simeq 1$. The existence of a di-muon resonance with these properties cannot be excluded because of the large hadronic uncertainties of the SM prediction for $B \rightarrow K^{(*)} \mu^{+} \mu^{-}$in the $m_{\mu \mu}^{2} \gtrsim 6 \mathrm{GeV}^{2}$ region (cf. [36,37]) and the unknown interference pattern between the $J / \psi$ and the SM short-distance contribution. By choosing the couplings in (1) to be $g_{L}^{s b} \simeq-10^{-8}, g_{V}^{\mu} \simeq 0.1$ and $g_{A}^{\mu} \simeq-0.44 g_{V}^{\mu}$, the discussed simplified model then does not only provide a solution to the flavor anomalies but also ameliorates the discrepancy observed in $a_{\mu}$. Other constraints that arise from $B_{s}-\bar{B}_{s}$ mixing, searches for $B \rightarrow$ $K^{(*)}+$ invisible [38-41], $B_{s} \rightarrow \mu^{+} \mu^{-}[42,43]$ and $Z \rightarrow 4 \mu$ $[44,45]$ as well as the precision measurements of $Z \mu \bar{\mu}$ couplings [46], are all satisfied for this choice of parameters.

\section{Z-BOSON LINE SHAPE}

We now consider the di-muon invariant mass spectrum as measured in DY production and study its distortions due to FSR of a light spin-1 resonance $V$. A representative diagram that contributes to $p p \rightarrow Z / \gamma^{*} \rightarrow \mu^{+} \mu^{-}+V$ is shown in Fig. 1. We calculate the $m_{\mu \mu}$ spectra with MADGRAPH5_AMC@NLO [47] and NNPDF23_LO_AS_0130_QED parton distribution functions [48], employing the DMSIMP implementation [49] of the $V \mu \bar{\mu}$ and $V \chi \bar{\chi}$ couplings in (1). The fiducial phase space in our Monte Carlo simulations is defined by requiring that the muon transverse momenta satisfy $p_{T, \mu}>25 \mathrm{GeV}$, the muon pseudorapidities obey $\left|\eta_{\mu}\right|<2.5$, and that $m_{\mu \mu}$ falls into the range 66-116 GeV.

In Fig. 2 we present our results for the di-muon invariant mass spectra for $p p$ collisions at a center-of-mass energy of

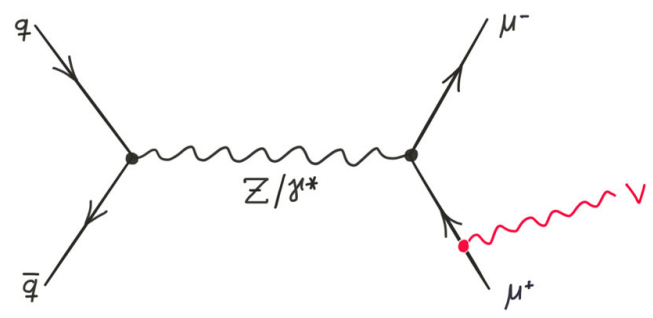

FIG. 1. An example of a Feynman diagram with FSR of the light mediator $V$ in the DY process $p p \rightarrow Z / \gamma^{*} \rightarrow \mu^{+} \mu^{-}$.

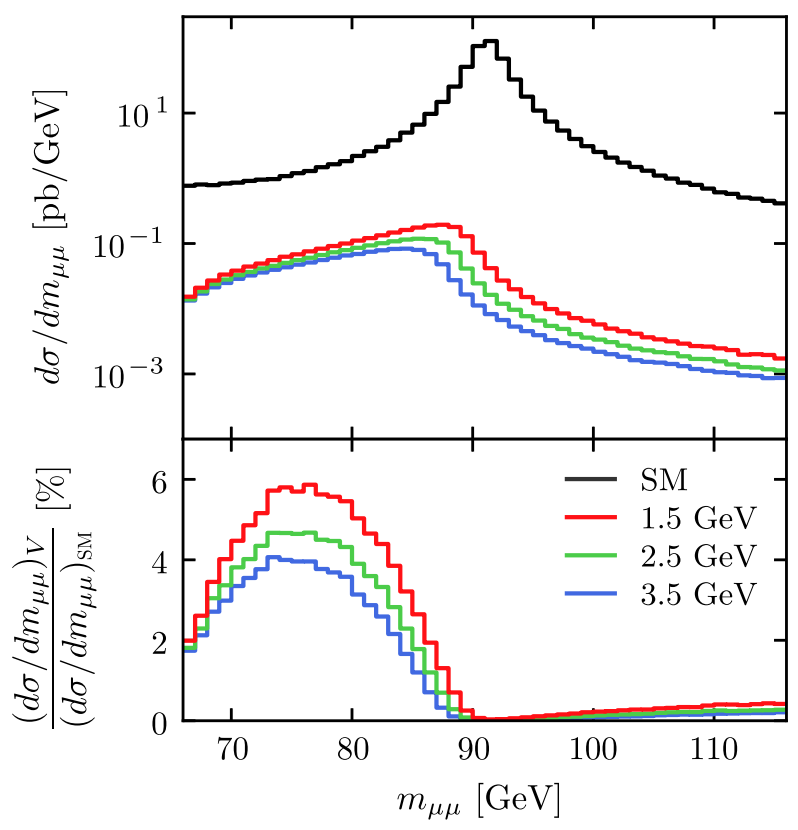

FIG. 2. Di-muon invariant mass distributions. The black curve represents the SM prediction, while the colored curves correspond to three different benchmark models with varying spin-1 resonance mass. For further details see the text.

$\sqrt{s}=13 \mathrm{TeV}$. All predictions are obtained at leading order in QCD. The three colored curves correspond to $g_{V}^{\mu}=0.1$ and $g_{A}^{\mu}=-4.4 \times 10^{-2}$ and mediator masses of 1.5, 2.5 and $3.5 \mathrm{GeV}$, respectively. For comparison, the SM prediction for the $Z$-boson line shape is depicted by the black curve. One observes that FSR of the spin-1 resonance leads to a pronounced radiation tail below $m_{Z} \simeq 91.2 \mathrm{GeV}$. This amounts to a relative correction to the SM Z-boson line shape of around $4 \%$ to $6 \%$ at $m_{\mu \mu} \simeq 75 \mathrm{GeV}$.

DY processes are a cornerstone of the SM physics program at the LHC (see e.g. [50-55] for recent ATLAS and CMS analyses) and a detailed understanding of the $Z$-boson line shape is a prerequisite for a precision measurement of the $W$-boson mass at the LHC [56]. Given its importance, a lot of effort has gone into measuring the $m_{\mu \mu}$ spectrum in the $Z$-peak region at the LHC and the experimental uncertainties have reached the few-percent level, making the $Z$-boson line shape a powerful observable to search for $\mathrm{GeV}$-mass di-muon spin-1 


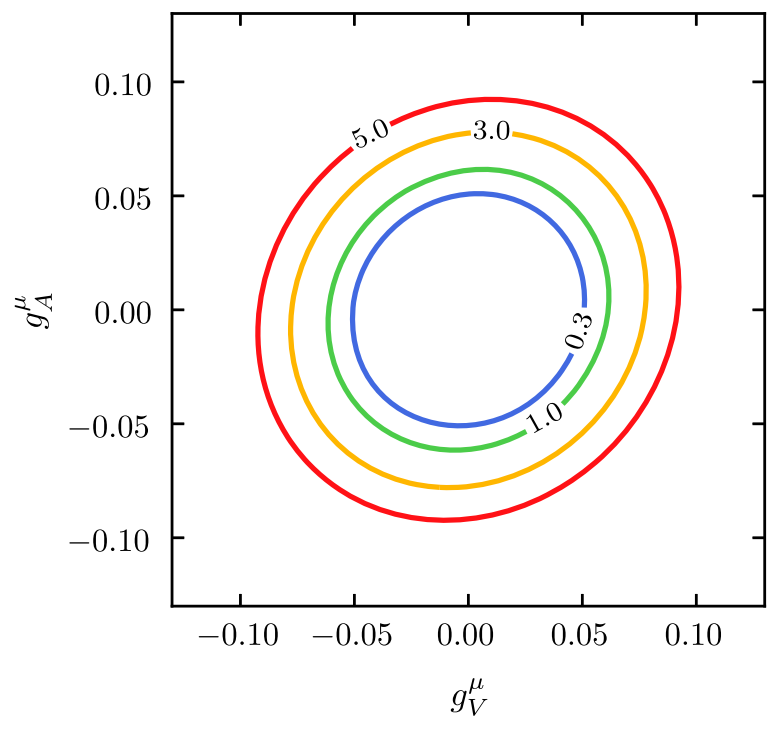

FIG. 3. Constraints in the $g_{V}^{\mu} g_{A}^{\mu}$ plane arising from DY production for different di-muon spin-1 resonance masses in units of $\mathrm{GeV}$. The contours correspond to $\Delta \chi^{2}=5.99$. See the text for additional details.

states. Besides the $m_{\mu \mu}$ spectra, other DY observables such as the fiducial cross section, the muon transverse momentum, the forward-backward asymmetry, or the distribution of the angular variable $\phi^{*}$ [57] are modified by both real and virtual corrections associated to (1). A comprehensive study of all effects in DY production related to a light spin1 di-lepton resonance is beyond the scope of this paper.

In our study we consider the ratio of the data to the SM prediction to perform a $\chi^{2}$ fit. In Fig. 2 of [51], the ATLAS collaboration provides the ratio of experimental data to the state-of-the-art theory prediction for the $m_{\mu \mu}$ line shape in the fiducial volume defined above. Assuming that the data is SM-like, we compute this ratio for different new-physics scenarios and perform a $\chi^{2}$ analysis. Radiative corrections of QCD and electroweak nature do not affect the ratio and are therefore neglected in the following. The ATLAS analysis is based on $3.2 \mathrm{fb}^{-1}$ integrated luminosity at $\sqrt{s}=13 \mathrm{TeV}$. The experimental statistical and systematic uncertainties are in the range of $1 \%$ to $2 \%$ and they are added in quadrature in our fit. The bin-to-bin correlations are neglected. ${ }^{3}$ We note that normalized distributions should be used in order to reduce the luminosity and systematic uncertainties.

In Fig. 3 we show the $\Delta \chi^{2}=5.99$ contours [corresponding to a 95\% confidence level (C.L.) for a Gaussian distribution] in the $g_{V}^{\mu}-g_{A}^{\mu}$ plane that follow from our $\chi^{2}$ analysis for different values of $m_{V}$. The parameter space

\footnotetext{
${ }^{3}$ In our analysis, we do not include luminosity uncertainties since they are highly correlated between bins and the correlation matrix is not given in [51]. Including a $2.1 \%$ uncorrelated luminosity uncertainty weakens the bound in Fig. 4 by around $10 \%$.
}
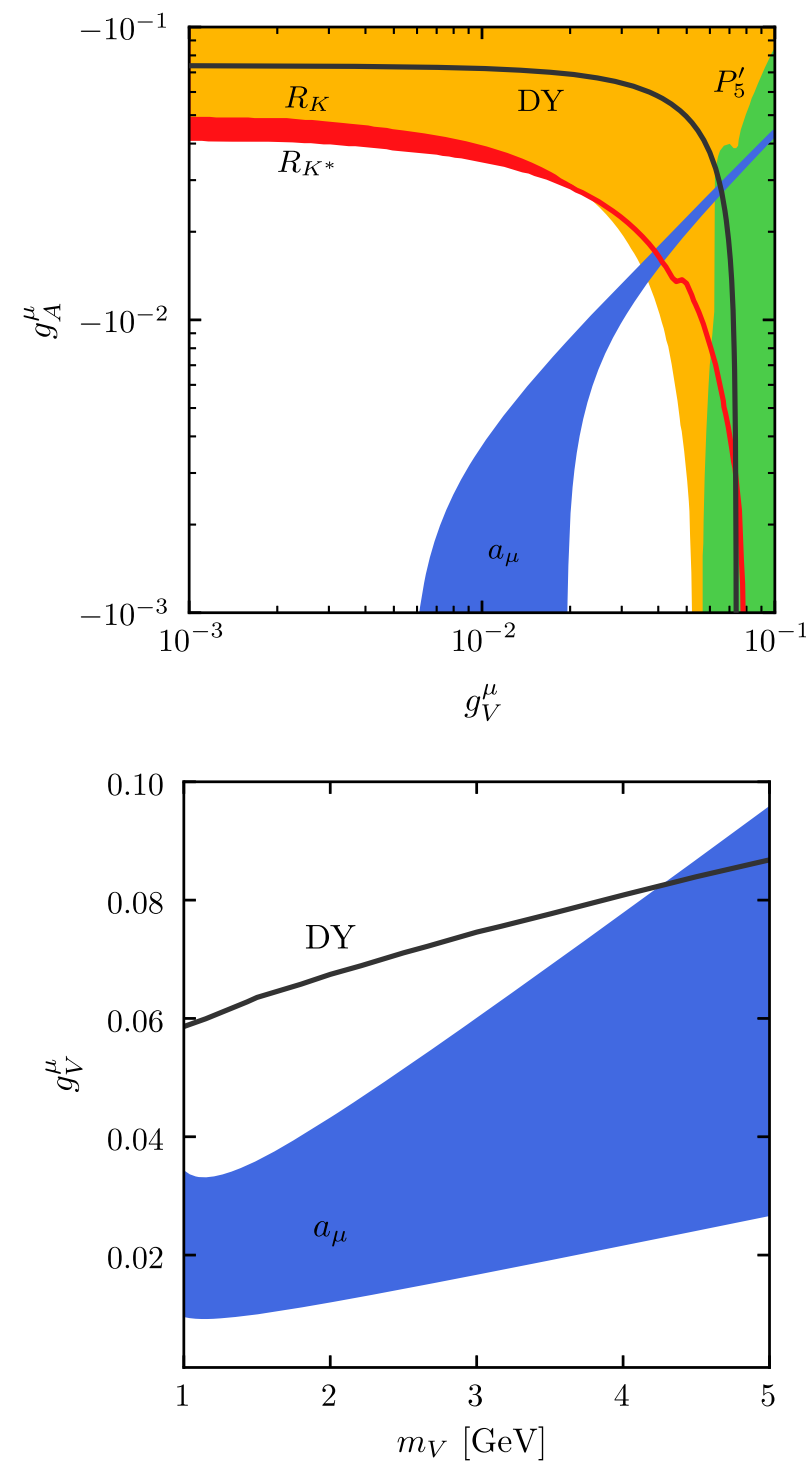

FIG. 4. Upper panel: Constraints in the $g_{V}^{\mu} g_{A}^{\mu}$ plane. The shown results were obtained for $m_{V}=2.5 \mathrm{GeV}$ and $g_{L}^{s b}=-1.5 \times 10^{-8}$. Lower panel: Constraints in the $m_{V^{-}} g_{V}^{\mu}$ plane assuming the coupling relation $g_{A}^{\mu}=0.41 g_{V}^{\mu}$. Consult the main text for further explanations.

outside the lines is disfavored for each individual mass. We find that for $m_{V} \in[1,5] \mathrm{GeV}$ the obtained $95 \%$ C.L. bounds can be approximately described by the inequality

$$
\sqrt{\left(g_{V}^{\mu}\right)^{2}+\left(g_{A}^{\mu}\right)^{2}} \lesssim 5.6 \times 10^{-2}\left(1+0.13 \frac{m_{V}}{1 \mathrm{GeV}}\right) .
$$

This approximate formula can be used to quickly assess the sensitivity of existing DY measurements on the coupling strength of GeV-mass di-muon spin-1 states.

The upper panel in Fig. 4 compares the 95\% C.L. constraint in the $g_{V}^{\mu}-g_{A}^{\mu}$ plane that derives from our fit to the $Z$-boson line shape for $m_{V}=2.5 \mathrm{GeV}$ (black) to the regions preferred by $P_{5}^{\prime}$ (green), $R_{K}$ (yellow) and $R_{K^{*}}$ (red) 
and $a_{\mu}$ (blue). The parameter space above and to the right of the black curve is excluded. In the case of the flavor observables the favored parameter space corresponds to the $\Delta \chi^{2}=4$ regions obtained in [22] for $g_{L}^{s b}=-1.5 \times 10^{-8}$, while in the case of $a_{\mu}$ we have employed the $3 \sigma$ bound $\Delta a_{\mu} \in[49,527] \times 10^{-11}$ [33]. From the panel it is evident that the model-independent constraint that arises from the DY data excludes parts of the parameter space favored by the $b \rightarrow s \ell^{+} \ell^{-}$anomalies. In particular, coupling choices that accommodate the deviation seen in $P_{5}^{\prime}$ are constrained. We now focus on the region of the $g_{V}^{\mu}-g_{A}^{\mu}$ plane in which the discrepancy between SM and data for $a_{\mu}$ is improved by the one-loop corrections due to the exchange of a light di-muon spin-1 resonance (cf. [58]),

$$
\Delta a_{\mu}=\frac{\left(g_{V}^{\mu}\right)^{2}-5\left(g_{A}^{\mu}\right)^{2}}{12 \pi^{2}} \frac{m_{\mu}^{2}}{m_{V}^{2}}+\mathcal{O}\left(m_{\mu}^{4} / m_{V}^{4}\right)
$$

In this region, we observe that our new constraint disfavors most of the parameter space that provides a simultaneous explanation of $P_{5}^{\prime}, R_{K}, R_{K^{*}}$ and $a_{\mu}$. Given the weak mass dependence of (2), we expect this conclusion to hold in the full range $m_{V} \in[2,3] \mathrm{GeV}$ of spin-1 resonance interpretations of the flavor anomalies.

In the lower panel of Fig. 4, we compare the 95\% C.L. bound in the $m_{V^{-}}-g_{V}^{\mu}$ plane following from measurements of the $m_{\mu \mu}$ spectrum in DY production (black) to the region favored by $a_{\mu}$ (blue). The shown results have been obtained for $g_{A}^{\mu}=0.41 g_{V}^{\mu}$. We see that our new DY constraint shrinks the allowed parameter space for such fine-tuned solutions of the $a_{\mu}$ anomaly for resonances heavier than about $4.2 \mathrm{GeV}$. Spin-1 resonance explanations of $a_{\mu}$ that do not rely on a cancellation in the combination $\left(g_{V}^{\mu}\right)^{2}-$ $5\left(g_{A}^{\mu}\right)^{2}$ of couplings, such as solutions with $g_{V}^{\mu} \neq 0$ and $g_{A}^{\mu}=0$, on the other hand, cannot be probed through $Z$-boson line shape measurements at present.

\section{CONCLUSIONS}

The main goal of this paper was to point out that precision measurements of DY production provide sensitive probes of light di-leptonic resonances. In view of the various deviations from SM predictions observed in rare semileptonic $B$ decays, we have applied our general observation to the case of $\mathrm{GeV}$-mass di-muon spin-1 resonances. Specifically, we have analyzed the distortions that FSR of such mediators imprints on the di-muon invariant mass spectrum as measured in $p p \rightarrow Z / \gamma^{*} \rightarrow$ $\mu^{+} \mu^{-}$at the LHC. For simplified-model realizations that allow one to qualitatively reproduce the $P_{5}^{\prime}, R_{K}, R_{K^{*}}$ and $a_{\mu}$ anomalies, we have found that the $Z$-boson line shape develops a pronounced radiative tail that amounts to a relative enhancement of $\mathcal{O}(5 \%)$ at $m_{\mu \mu} \simeq 75 \mathrm{GeV}$ compared to the SM prediction.

Motivated by this finding we have derived modelindependent bounds on the muon couplings of spin-1 mediators using DY data from LHC run II. Our analysis shows that the existing precision measurements of DY production put nontrivial constraints on the parameter space of light di-muon resonance models [22] that aim at explaining the tensions seen in rare semileptonic $B$ decays. In particular, they disfavor almost all model realizations that can simultaneously accommodate the $P_{5}^{\prime}, R_{K}, R_{K^{*}}$ and $a_{\mu}$ anomalies. Considering $a_{\mu}$ alone, we found instead that present $Z$-boson line shape fits can only probe fine-tuned $\mathrm{GeV}$-mass explanations of the anomaly with $\left|g_{A}^{\mu}\right| \simeq 0.44\left|g_{V}^{\mu}\right|$. Since the data set used to derive the constraints contains only $3.2 \mathrm{fb}^{-1}$ of integrated luminosity collected at $\sqrt{s}=13 \mathrm{TeV}$, future analyses performed at LHC run II and beyond are expected to strengthen the obtained bounds in case no deviations from the $m_{\mu \mu}$ spectrum as predicted in the SM are found.

While in our work we have focused our attention on light di-muon spin-1 resonances, precision measurements of the kinematic distributions of the final-state leptons in $p p \rightarrow Z / \gamma^{*} \rightarrow \ell^{+} \ell^{-}$can also be used to search for and to constrain mediators preferentially coupling to electron pairs and/or of different spin. A dedicated study of the DY constraints on alternative light di-lepton resonance scenarios, while beyond the scope of this paper, thus seems to be a worthwhile exercise.

\section{ACKNOWLEDGMENTS}

We thank David Straub for useful discussions concerning [22]. The work of F. B. is supported by the Science and Technology Facilities Council (STFC). U. H. acknowledges the support of the CERN Theoretical Physics Department.
[1] S. Descotes-Genon, J. Matias, M. Ramon, and J. Virto, J. High Energy Phys. 01 (2013) 048.

[2] R. Aaij et al. (LHCb Collaboration), Phys. Rev. Lett. 111, 191801 (2013).
[3] R. Aaij et al. (LHCb Collaboration), J. High Energy Phys. 02 (2016) 104.

[4] S. Wehle et al. (Belle Collaboration), Phys. Rev. Lett. 118, 111801 (2017). 
[5] ATLAS Collaboration, Technical Report No. ATLASCONF-2017-023, CERN, Geneva, 2017.

[6] R. Aaij et al. (LHCb Collaboration), J. High Energy Phys. 06 (2014) 133.

[7] R. Aaij et al. (LHCb Collaboration), J. High Energy Phys. 11 (2016) 047.

[8] R. Aaij et al. (LHCb Collaboration), J. High Energy Phys. 09 (2015) 179.

[9] G. Hiller and F. Krüger, Phys. Rev. D 69, 074020 (2004).

[10] R. Aaij et al. (LHCb Collaboration), Phys. Rev. Lett. 113, 151601 (2014).

[11] R. Aaij et al. (LHCb Collaboration), J. High Energy Phys. 08 (2017) 55.

[12] B. Capdevila, A. Crivellin, S. Descotes-Genon, J. Matias, and J. Virto, arXiv:1704.05340.

[13] W. Altmannshofer, P. Stangl, and D. M. Straub, arXiv:1704 .05435 .

[14] G. D’Amico, M. Nardecchia, P. Panci, F. Sannino, A. Strumia, R. Torre, and A. Urbano, arXiv:1704.05438.

[15] G. Hiller and I. Nisandzic, arXiv:1704.05444.

[16] L.-S. Geng, B. Grinstein, S. Jäger, J. M. Camalich, X.-L. Ren, and R.-X. Shi, arXiv:1704.05446.

[17] M. Ciuchini, A. M. Coutinho, M. Fedele, E. Franco, A. Paul, L. Silvestrini, and M. Valli, arXiv:1704.05447.

[18] A. Celis, J. Fuentes-Martin, A. Vicente, and J. Virto, arXiv: 1704.05672

[19] D. Becirevic and O. Sumensari, arXiv:1704.05835.

[20] Y. Cai, J. Gargalionis, M. A. Schmidt, and R. R. Volkas, arXiv: 1704.05849.

[21] J. F. Kamenik, Y. Soreq, and J. Zupan, arXiv:1704.06005.

[22] F. Sala and D. M. Straub, arXiv:1704.06188.

[23] D. Ghosh, arXiv:1704.06240.

[24] S. Di Chiara, A. Fowlie, S. Fraser, C. Marzo, L. Marzola, M. Raidal, and C. Spethmann, arXiv:1704.06200.

[25] A. K. Alok, D. Kumar, J. Kumar, and R. Sharma, arXiv: 1704.07347.

[26] A. K. Alok, B. Bhattacharya, A. Datta, D. Kumar, J. Kumar, and D. London, arXiv:1704.07397.

[27] W. Wang and S. Zhao, arXiv:1704.08168.

[28] A. Greljo and D. Marzocca, arXiv:1704.09015.

[29] C. Bonilla, T. Modak, R. Srivastava, and J. W. F. Valle, arXiv:1705.00915.

[30] F. Feruglio, P. Paradisi, and A. Pattori, arXiv:1705.00929.

[31] K. Fuyuto, W.-S. Hou, and M. Kohda, Phys. Rev. D 93, 054021 (2016).

[32] A. Datta, J. Liao, and D. Marfatia, Phys. Lett. B 768, 265 (2017)

[33] C. Patrignani et al. (Particle Data Group Collaboration), Chin. Phys. C 40, 100001, (2016).
[34] P. J. Fox, J. Liu, D. Tucker-Smith, and N. Weiner, Phys. Rev. D 84, 115006 (2011).

[35] C. D. Carone, Phys. Lett. B 721, 118 (2013).

[36] A. Khodjamirian, T. Mannel, A. A. Pivovarov, and Y. M. Wang, J. High Energy Phys. 09 (2010) 089.

[37] J. Lyon and R. Zwicky, arXiv:1406.0566.

[38] P. del Amo Sanchez et al. (BABAR Collaboration), Phys. Rev. D 82, 112002 (2010).

[39] O. Lutz et al. (Belle Collaboration), Phys. Rev. D 87, 111103 (2013).

[40] J. P. Lees et al. (BABAR Collaboration), Phys. Rev. D 87, 112005 (2013).

[41] J. Grygier et al. (Belle Collaboration), arXiv:1702.03224.

[42] V. Khachatryan et al. (LHCb and CMS Collaborations), Nature (London) 522, 68 (2015).

[43] R. Aaij et al. (LHCb Collaboration), Phys. Rev. Lett. 118, 191801 (2017).

[44] S. Chatrchyan et al. (CMS Collaboration), J. High Energy Phys. 12 (2012) 034.

[45] G. Aad et al. (ATLAS Collaboration), Phys. Rev. Lett. 112, 231806 (2014).

[46] S. Schael et al. (SLD Electroweak Group, DELPHI, ALEPH, SLD, SLD Heavy Flavour Group, OPAL, LEP Electroweak Working Group, L3 Collaborations), Phys. Rep. 427, 257 (2006).

[47] J. Alwall, R. Frederix, S. Frixione, V. Hirschi, F. Maltoni, O. Mattelaer, H. S. Shao, T. Stelzer, P. Torrielli, and M. Zaro, J. High Energy Phys. 07 (2014) 079.

[48] R. D. Ball, V. Bertone, S. Carrazza, L. Del Debbio, S. Forte, A. Guffanti, N. P. Hartland, and J. Rojo (NNPDF Collaboration), Nucl. Phys. B877, 290 (2013).

[49] M. Backovic, M. Krämer, F. Maltoni, A. Martini, K. Mawatari, and M. Pellen, Eur. Phys. J. C 75, 482 (2015).

[50] M. Aaboud et al. (ATLAS Collaboration), Eur. Phys. J. C 77, 367 (2017).

[51] M. Aaboud et al. (ATLAS Collaboration), J. High Energy Phys. 02 (2017) 117.

[52] M. Aaboud et al. (ATLAS Collaboration), Eur. Phys. J. C 77, 361 (2017).

[53] CMS Collaboration, Technical Report No. CMS-PAS-SMP15-002, CERN, Geneva, 2016.

[54] CMS Collaboration, Technical Report No. CMS-PAS-SMP15-010, CERN, Geneva, 2015.

[55] CMS Collaboration, Technical Report No. CMS-PAS-SMP16-009, CERN, Geneva, 2016.

[56] M. Aaboud et al. (ATLAS Collaboration), arXiv:1701 .07240 .

[57] A. Banfi, S. Redford, M. Vesterinen, P. Waller, and T. R. Wyatt, Eur. Phys. J. C 71, 1600 (2011).

[58] F. Jegerlehner and A. Nyffeler, Phys. Rep. 477, 1 (2009). 